\title{
Transcriptome analysis of hot pepper plants identifies waterlogging resistance related genes
}

\author{
Yuping Zhang ${ }^{1}$, Lijun $\mathrm{Ou}^{2}$, Ji Zhao ${ }^{2}$, Zhoubin $\mathrm{Liu}^{3}$, and Xuefeng $\mathrm{Li}^{2 *}$ \\ ${ }^{1}$ Hunan Agricultural University, College of Resources and Environment, Changsha, 410128, China. \\ ${ }^{2}$ Hunan Academy of Agricultural Science, Institute of Vegetable Research, Changsha, Hunan 410125, China. \\ "Corresponding author: Xuefeng Li (1xf276@126.com). \\ ${ }^{3}$ Longping Branch, Graduate School of Hunan University, Changsha, Hunan 410006, China.
}

Received: 29 September 2018; Accepted: 17 January 2019; doi:10.4067/S0718-58392019000200296

\begin{abstract}
Hot pepper (Capsicum annuum L.) is one of the most important vegetable crops in China, but floods bring substantial decreases in production over the past several decades. In order to investigate the mechanisms of waterlogging resistance in mutant hot pepper, we measured the agronomic traits of mutant and wild-type, as well as the activities of root antioxidant enzymes and the contents of osmotic regulation substance. At the same time, we did transcriptome sequencing on the plant roots, and screened for differentially expressed genes between mutant and wild-type. The results showed that, under waterlogging stress, the mutants could grow normally, and the activities of their superoxide dismutase, peroxidase, catalase, and glutathione reductase were significantly increased, as well as the contents of proline and soluble sugar. The accumulation of malondialdehyde and hydroxyl radical in mutants was significantly reduced. Among the 61 differentially expressed genes from transcriptome analysis, 24 genes were up-regulated and 37 genes were down-regulated in mutants. After functional analysis, we found 8 genes related to the metabolism of endogenous hormone and protective enzymes, among which, auxin-induced protein related gene cap.ARATH, ethylene response related gene Cap.RAP2, MYB family related gene Cap.MYB1R1, and the 4 genes related to peroxidase Cap.POD, were significantly up-regulated in mutants, while the Capana01g001329 gene was down-regulated. These results suggest that under waterlogging stress, the mutant could enhance its resistance to waterlogging by regulating the genes involved in metabolism of endogenous hormones and protective enzymes. This study provides the scientific basis for elucidating the gene regulation network of mutant pepper under waterlogging stress.
\end{abstract}

Key words: Capsicum annuи, gene, hot pepper, mutant, transcriptomic data, waterlogging.

\section{INTRODUCTION}

Pepper (Capsicum annuиm L.) belongs to Solanaceae family, Capsicum genus. It is an annual herb or limited perennial herb. Pepper originates from the tropical areas in middle Latin America, and is widely used in vegetables and spices, as well as in other applications such as medicines, natural colors, cosmetics and ornamental plants. Also, pepper is one of the most important vegetable species in China, with the annual planting area of more than 1.3 million ha (Zou, 2009). Pepper has strong drought tolerance (Ou et al., 2012), but its ability to resist waterlogging is weak (Ou et al., 2011). It is easy to catch virus disease during rainy seasons, resulting in serious flower and fruit falling. Sometimes, too many fallen leaves even cause the plant death. In China, $60 \%-70 \%$ of the peppers are growing in open field. In many pepper production areas, the agricultural infrastructure such as irrigation is incomplete, so the peppers are often subjected to waterlogging and other disasters, bringing great losses to pepper production and quality. Moreover, it is very difficult to find waterlogging-resistant peppers in routine breeding, so the discovery or accumulation of waterlogging resistant genes is nearly impossible. 
The important ways to improve crop waterlogging resistance are discovering new waterlogging resistant genes and introducing them into crop genome and using molecular markers to select and accumulate resistant genes (Valliyodan et al., 2017). Zou et al. (2013) examined the gene expression profiles of the roots from waterlogging resistant rape seedlings after $12 \mathrm{~h}$ of waterlogging treatment, and found 4432 differentially expressed genes. They analyzed the 200 highly expressed genes, and found 144 of them were down-regulated under waterlogging stress. Moreover, they identified 7 genes encoding for ethylene responsive factors (ERF) were regulated upon waterlogging stress, and 5 of them, including ERF2, ERF4, ERF7, ERF11 and ERF54, were induced by stress. Lee et al. (2014) analyzed the gene expression profiles of rapeseed leaves after 36 and $72 \mathrm{~h}$ of waterlogging stress, and found that compared to control, the photosynthesis related genes were down-regulated, and the genes related to scavenging reactive oxygen, protein, starch and lipid degradation were up-regulated.

An effective way to improve plant qualities is mutagenesis treatment via physical or chemical factors, which has been widely used in crops, gardening, flowers, etc. Ethyl methanesulfonate (EMS) mutagenesis is one of the commonly used methods to obtain mutants manually, and is more specific compared to other mutagenesis methods. The EMS target is DNA base, causing base changes such as substitution and reversion. Since the mutants generated by EMS usually only have alterations on individual bases, they have simple backgrounds as compared to the extreme cases obtained from conventional screening, thereby they can reveal the related regulatory networks in a better way. There have been studies using the mutants obtained from EMS mutagenesis to investigate the salt tolerance (Pei et al., 2017), resistance to disease (Wu et al., 2008; Xu et al., 2017a), low temperature tolerance (Jin et al., 2003) and drought tolerance (Zhang et al., 2011) in rice, alfalfa, Chinese wolfberry, bilberry and other species. So far, due to the lack of mutants, the selection of waterlogging resistant pepper and gene analysis on related functions mainly depend on conventional methods, primarily focusing on phenotypical, physiological and biochemical parameters. In 2013, our group mutated 'S15' via EMS, and found a mutant with strong waterlogging resistance from screening, named 'RW15'. In this paper, we treated the mutant and wild-type with waterlogging stress, and compared their differences in basic agronomic traits, antioxidant enzyme activities, and the content of osmotic regulation substance. We also sequenced the transcriptomes of their roots, and analyzed the differences in gene expression and regulation, in order to find the important genes involved in waterlogging stress response and elucidate the mechanisms of waterlogging resistance in mutants.

\section{MATERIALS AND METHODS}

\section{Plant materials and stress conditions}

In 2013, we obtained the $\mathbf{M}_{1}$ generation from EMS processed 'S15', and then acquired the $\mathbf{M}_{2}$ generation after selfcrossing. After conducting waterlogging screening on $\mathrm{M}_{2}$ generation, we obtained a waterlogging resistant mutant strain, and self-crossed it to get $\mathrm{M}_{3}$ seeds. The seeds of $\mathrm{M}_{3}$ and wild-type (WT) peppers were germinated and planted in seedling tray. At the 5-leaf-1-heart phase, the robust seedlings with consistent growth rate were planted in $9 \mathrm{~cm} \times 9 \mathrm{~cm}$ nursery pots, 1 seedling per pot. The pots were filled with soil until $1 \mathrm{~cm}$ to the edge, and the soil contained $60 \%$ farmland soil, $20 \%$ cow dung compost, and $20 \%$ river mud. When seedlings grew to the 6-leaf-1-heart period, they were subjected to waterlogging stress using dual-pot method. The nursery pots were put into a $15 \mathrm{~cm} \times 40 \mathrm{~cm}$ larger pot, each larger pot included 6 pots ( 3 mutants, $3 \mathrm{WT}$ ). The water level at $2 \mathrm{~cm}$ above nursery pot surface was considered waterlogging. The water was supplemented every other day to maintain the flooding level, and the waterlogging treatment was repeated for three times. Ten days after the treatment, leaves and roots were washed with clean water, wiped, and quickly frozen with liquid nitrogen. The samples were stored in $-80{ }^{\circ} \mathrm{C}$ freezer for future use.

\section{Agronomic characterization}

The height and root length of randomly selected pepper plants were measured with a ruler. The dry weight and fresh weight of over-ground part, underground part and the entire plant were recorded. Then the plants were killed at $105^{\circ} \mathrm{C}$ for $30 \mathrm{~min}$, and dried at $75^{\circ} \mathrm{C}$ until constant weight.

\section{Measurements of enzyme activity and plant inclusions}

Proline content was measured by the acid ninhydrin method. The fresh leaves were washed and dried with paper towel. 
Samples of leaves $(0.5 \mathrm{~g})$ were cut into pieces, mixed with $5 \mathrm{~mL} 3 \%$ sulfosalicylic acid solution in a large tube, and boiled for $10 \mathrm{~min}$ under shaking. The extract was filtered with a funnel into a clean tube after cooling down, $2 \mathrm{~mL}$ filtered extract was mixed with $2 \mathrm{~mL}$ acetic acid and $2 \mathrm{~mL}$ acid ninhydrin, and boiled for $30 \mathrm{~min}$ after sealed with plastic wrap. After cooling down, $4 \mathrm{~mL}$ toluene was added into the tube and fully oscillated. The upper red solution was collected and its absorption at $520 \mathrm{~nm}$ was measured using toluene as reference. Proline content $\left[\mathrm{x}\left(\mu \mathrm{g} \mathrm{mL}^{-1}\right)\right]$ in the $2 \mathrm{~mL}$ tested samples was obtained from the standard curve and the proline concentration in fresh leaves was calculated based on the following formula: Proline content $\left(\mu \mathrm{g} \mathrm{g}^{-1}\right)=(\mathrm{x} \times 5 / 2) /$ fresh leaf weight $(\mathrm{g})$.

Anthrone method was used to measure soluble sugar content. They were measured with anthrone colorimetry. Samples of leaves $(0.1 \mathrm{~g})$ were placed in a ground glass weighing bottle, mixed with $20 \mathrm{~mL}$ distilled water, and extracted twice in boiling water for $30 \mathrm{~min}$. The extract was filtered into a $50 \mathrm{~mL}$ volumetric flask, and the weighing bottle and residues were washed repeatedly before being filled to constant volume. Sample extract $(0.5 \mathrm{~mL})$ was mixed thoroughly with 1.5 $\mathrm{mL}$ distilled water, $0.5 \mathrm{~mL}$ anthrone ethyl acetate solution and $5 \mathrm{~mL}$ concentrated sulfuric acid, before being immediately placed in boiling water bath for $1 \mathrm{~min}$. The heated sample extract was cooled naturally to room temperature to determine the absorbance at $630 \mathrm{~nm}$. The final soluble sugar contents were calculated from the standard curve: soluble sugar $\left(\mu \mathrm{g} \mathrm{g}^{-1}\right.$ $\mathrm{FW})=[$ (corresponding sucrose content from standard curve $(\mu \mathrm{g}) \times$ total extract volume $(\mathrm{mL})] /[$ (measurement volume $(\mathrm{mL}) \times$ fresh weight of the sample $(\mathrm{g})]$.

The content of malondialdehyde (MDA) was determined by thiobarbituric acid (TBA method) by commercial kits (Nanjing Jiancheng Bioengineering Institute, Nanjing China). Samples of leaves $(0.1 \mathrm{~g})$ were thoroughly ground with a cold mortar and pestle in an ice bath. The grinding medium was $4 \mathrm{~mL}$ saline, plus homogenizing glass beads. The homogenate was centrifuged for $10 \mathrm{~min}$ at $2500 \mathrm{rpm}$ and $25^{\circ} \mathrm{C}$. The supernatant referred was crude enzyme extract and used to determination. The absorbance of the reaction mixture was determined by using a spectrophotometer (UV-1780, Shimadzu, Kyoto, Japan).

The colorimetric assay was used to measure the contents of peroxidase (POD), catalase (CAT), superoxide dismutase (SOD), glutathione reductase (GS) and hydroxyl free radicals ( $\mathrm{OH}$ ) (the kit was purchased from Nanjing Jiancheng Bioengineering Institute, Nanjing Shi, China) (Yang et al., 2016). A sample of leaves $(0.1 \mathrm{~g})$ without midrib were thoroughly ground with a cold mortar and pestle in an ice bath. The grinding medium was $4 \mathrm{~mL}$ saline, plus homogenizing glass beads. The homogenate was centrifuged for $10 \mathrm{~min}$ at $2500 \mathrm{rpm}$ and $4{ }^{\circ} \mathrm{C}$. The supernatant referred was crude enzyme extract and used to determination. The absorbance of the reaction mixture was determined by using a UV-1780 spectrophotometry (Shimadzu, Japan).

\section{RNA Extraction, library preparation and sequencing}

Total RNA was extracted from tissues using TRI Reagent (Sigma Life Science, St. Louis, Missouri, USA), according to manufacturer's instructions. RNA quality was checked by RNase-free agarose gel electrophoresis to avoid possible degradation and contamination, and then verified using Agilent 2100 Bio-analyzer (Agilent Technologies, Santa Clara, California, USA). Next, Poly (A) mRNA was isolated using oligo-dT beads (Qiagen, Hilden, Germany), and then broken into short fragments by adding fragmentation buffer. First-strand cDNA was synthesized using random hexamer-primed reverse transcription, followed by the synthesis of the second-strand cDNA using RNase H and DNA polymerase I. The cDNA fragments were purified using a QIA quick PCR extraction kit, and then washed with EB buffer for end reparation poly (A) addition and ligated to sequencing adapters. Following agarose gel electrophoresis and extraction of cDNA from gels, the cDNA fragments were purified and enriched by PCR to construct the final cDNA library, which was then sequenced on the sequencing platform (HiSeq X Ten, Illumina, San Diego, California, USA) using the paired-end technology. Three biological replicates were performed for each line, thus six libraries were generated and sequenced.

\section{Transcriptome analysis}

Raw reads were filtered to remove low quality sequences (there were more than $50 \%$ bases with quality lower than 20 in one sequence), reads with more than $5 \% \mathrm{~N}$ bases (bases unknown) and reads containing adaptor sequences through the Perl program. Then the clean reads were mapped to the tomato reference genome using TopHat2 (http://ccb.jhu.edu/ software/tophat), with the following parameters: -min-intron-length 20 -max-intron-length 10000 -read-mismatches 1. The differentially expressed genes (DEGs) were identified using the $\mathrm{R}$ package edgeR ( $\mathrm{R}$ Foundation for Statistical 
Computing, Vienna, Austria). The expression level of each gene was calculated and normalized to FPKM (fragments per kilobase of transcript per million fragments mapped). The false discovery rate (FDR) was used to determine the threshold of the P-value in multiple tests. In our study, the FDR $<0.05$ and fold change $>2$ were used as significance cut-offs of the gene expression differences. The DEGs were used for Gene Ontology (GO) and KEGG (Kyoto Encyclopedia of Genes and Genomes) enrichment analyses according to Tan et al. (2018); GO terms with corrected P-value $<0.05$ and KEGG pathways with $\mathrm{P}$-value $<0.05$ were considered significantly enriched by differential expressed genes.

\section{Quantitative real-time PCR}

The total root RNA was extracted using TaKaRa MiniBEST Plant RNA Extraction Kit (Takara Bio, Kusatsu, Shiga, Japan), followed by reverse transcription to obtain a total of $200 \mathrm{ng}$ cDNA. qRT-PCR based on SYBR method was used to validate the DEGs (FastStart Essential DNA Green Master, Roche, Basel, Switzerland), CpAction was used as the internal reference gene (Liu et al., 2017). The qPCR primers (Table 1) were synthesized by Shanghai Biotech (Shanghai, China). PCR reaction system was $20 \mu \mathrm{L}$ : Master mix $10 \mu \mathrm{L}$, forward and reverse primers $0.5 \mu \mathrm{L}$ each, 10 times diluted cDNA 5.0 $\mu \mathrm{L}$, $\mathrm{H}_{2} \mathrm{O} 4 \mu \mathrm{L}$. The qPCR cycle was: $95^{\circ} \mathrm{C}$ for $10 \mathrm{~min}, 95^{\circ} \mathrm{C} 15 \mathrm{~s}, 60{ }^{\circ} \mathrm{C} 30 \mathrm{~s}, 72{ }^{\circ} \mathrm{C} 15 \mathrm{~s}$ for 45 cycles.

\section{Data analysis}

SPSS17.0 (IBM, Armonk, New York, USA) was used to conduct one-way ANOVA. LSD and Duncan's method were used for pairwise comparisons. $\mathrm{P}<0.05$ was considered significant.

\section{RESULTS}

\section{Phenotypic evaluation of experimental materials}

Under waterlogging stress, root length, plant height, fresh and dry weights of entire plant were all affected. Moreover, there were significant differences between mutant and WT after waterlogging treatment. The root length, plant height, fresh weight and dry weight of the mutants were $78.30 \%, 17.68 \%, 83.84 \%$ and $105.19 \%$ higher than WT, respectively (Table 2).

Table 1. Information of primer sequences.

\begin{tabular}{ll}
\hline Gene ID-accession & \multicolumn{1}{c}{ Primer sequence } \\
\hline Capana04g001523 & F:TTCTTGTTACTCTGCTCAA \\
& R:CGCTGGATGAATTGTAGTTC \\
Capana08g000001 & F:TCAGCAAGTCGTCATCAA \\
& R:TTCACTCCATTCACAGCATA \\
Capana01g001329 & R:TGCCAGCCACAATCTTAG \\
& F:TTGACAATAGCGGAAGGAT \\
Capana12g002272 & R:GAATGGAGAAGGAAGATTGTC \\
& F:TCAACAAGGAGGTTCTACTG \\
Capana02g003649 & R:GACAGCACAACATTATCCAA \\
& F:TCACGACTCTTACCTTGTAG \\
Capana03g002143 & R:TCCAACTTCCAACGCATT \\
& F:TTGAGGTGATTGATGCTGTA \\
Capana12g002274 & R:GAATGGAGAAGGAAGATTGTC \\
& F:ATGGACAGAGGAGGAACA \\
Capana05g002019 & R:AGATTGACGGTGGTGATG \\
CpAction & F:CCACCTCTTCACTCTCTGCTCT \\
& R:ACTAGGAAAAACAGCCCTTGGT \\
\hline
\end{tabular}

Table 2. Agronomic traits of pepper under waterlogging stress.

\begin{tabular}{|c|c|c|c|c|}
\hline & Root length & Plant height & Fresh weight & Dry weight \\
\hline & — & - & & 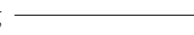 \\
\hline Wild-type & $4.47 \pm 0.15 b$ & $12.67 \pm 0.28 b$ & $1.12 \pm 0.03 b$ & $0.15 \pm 0.0011 b$ \\
\hline Mutant & $7.97 \pm 0.32 \mathrm{a}$ & $14.91 \pm 0.33 \mathrm{a}$ & $2.07 \pm 0.05 \mathrm{a}$ & $0.31 \pm 0.0026 \mathrm{a}$ \\
\hline
\end{tabular}

Each value is the mean \pm standard deviation $(S D, n=6)$. The effects are significant at $p<0.05$ with a one-way ANOVA. 


\section{Physiological response of pepper to waterlogging stress}

In addition, under waterlogging stress, the antioxidant enzymes in mutants showed higher activities: SOD, POD, CAT and GR enzymes of mutants were $90.61 \%, 77.92 \%, 43.05 \%$ and $106.67 \%$ more active than WT, respectively (Table 3). Meanwhile, the contents of proline and soluble sugar in mutants were $25.53 \%$ and $42.08 \%$ higher than WT (Table 4). And the contents of MDA and ${ }^{\circ} \mathrm{OH}$ were reduced in mutants by $47.54 \%$ and $68.72 \%$ compared to WT (Table 4).

\section{RNA-seq analysis}

Transcriptome sequencing was performed by Hiseq $X$ ten. After eliminating the joint and low-quality sequences, we obtained 211991416 clean and high-quality sequences with a total of $31.79 \mathrm{~Gb}$ data. Among these sequences, 1666942 723 of them were uniquely matched to the pepper genome. The percentage of Q30 bases were $89.67 \%-90.64 \%$, and the average GC content was about $45 \%$, indicating that the quality of transcriptome sequencing was relatively high, with good reproducibility between different replicates, which satisfied the criteria for further bioinformatic analysis (Table 5).

\section{GO functional annotation enrichment analysis}

Under waterlogging stress, 61 DEGs were detected between mutant and wild-type. Among them, 37 DEGs were downregulated in mutant, and 24 DEGs were up-regulated (Figure 1). After conducting the GO functional annotation analysis on differentially expressed genes, we found that these genes were concentrated in three major categories, biological processes, molecular functions and cell components (Figure 2), which included 6, 5 and 5 functional classes, respectively. The percentages of differential genes in these three categories were $45 \%, 35 \%$, and $20 \%$, respectively, Mutant and wild type had the largest differentially genes in single organism processes, antioxidant activity and membrane of this three categories, respectively, which indicated that most DEGs were significantly related to some of these biological functions. Indicating that most of these genes were significantly associated with some biological functions.

Table 3. Activities of antioxidant enzymes of pepper under waterlogging.

\begin{tabular}{lcrrc}
\hline & \multicolumn{1}{c}{ SOD } & POD & CAT & GR \\
\cline { 2 - 4 } & & & & $\mathrm{U} \mathrm{g} \mathrm{m}^{-1}$ prot \\
Wild-type & $64.85 \pm 5.36 \mathrm{~b}$ & $96.69 \pm 10.25 \mathrm{~b}$ & $38.91 \pm 3.62 \mathrm{~b}$ & $0.15 \pm 0.01 \mathrm{~b}$ \\
Mutant & $123.61 \pm 10.39 \mathrm{a}$ & $172.03 \pm 18.74 \mathrm{a}$ & $55.66 \pm 4.89 \mathrm{a}$ & $0.31 \pm 0.02 \mathrm{a}$ \\
\hline
\end{tabular}

Each value is the mean \pm standard deviation $(S D, n=6)$. The effects are significant at $p<0.05$ with a one-way ANOVA.

SOD: Superoxide dismutase; POD: peroxidase; CAT: catalase; GR: glutathione reductase.

Table 4. Contents of osmotic substances and oxidation products of pepper under waterlogging.

\begin{tabular}{lcccc}
\hline & PRO & Soluble sugar & MDA & OH \\
\hline$\mu \mathrm{g} \mathrm{g}^{-1} \mathrm{FW}$ & $\mathrm{mg} \mathrm{g}^{-1} \mathrm{FW}$ & nmol mg & prot & $\mathrm{U} \mathrm{mg}^{-1} \mathrm{prot}^{-1}$ \\
Wild-type & $45.95 \pm 3.98 \mathrm{~b}$ & $23.17 \pm 1.92 \mathrm{~b}$ & $71.24 \pm 6.65 \mathrm{a}$ & $30.75 \pm 3.25 \mathrm{a}$ \\
Mutant & $57.68 \pm 4.85 \mathrm{a}$ & $32.92 \pm 2.84 \mathrm{a}$ & $37.37 \pm 3.25 \mathrm{~b}$ & $9.62 \pm 0.87 \mathrm{~b}$ \\
\hline
\end{tabular}

Each value is the mean \pm standard deviation $(\mathrm{SD}, \mathrm{n}=6)$. The effects are significant at $\mathrm{p}<0.05$ with a one-way ANOVA.

PRO: Proline; MDA: malondialdehyde; $\mathrm{OH}$ : hydroxyl free radical.

Table 5. RNAseq data statistics.

\begin{tabular}{lccccccc}
\hline \multirow{2}{*}{ Group } & Sample ID & $\begin{array}{c}\text { Number of } \\
\text { input reads }\end{array}$ & $\begin{array}{c}\text { Input base } \\
(\mathrm{Gb})\end{array}$ & $\begin{array}{c}\text { Uniquely } \\
\text { mapped reads }\end{array}$ & $\begin{array}{c}\text { GC } \\
\text { content \% }\end{array}$ & $\begin{array}{c}\text { Bases } \\
\text { Q20\% }\end{array}$ & $\begin{array}{c}\text { Bases } \\
\text { Q30\% }\end{array}$ \\
\hline Wild-type & 7R4351Q2B361PB & 35752891 & 5.36 & 28219648 & 45.92 & 95.85 & 90.60 \\
& 7R4352Q2B362PB & 33306020 & 5.00 & 27558517 & 45.43 & 95.36 & 89.68 \\
& 7R4355Q2B363PB & 36496662 & 5.47 & 28154324 & 46.41 & 95.26 & 89.44 \\
\multirow{3}{*}{ Mutant } & 7R4344Q2B358PA & 35821154 & 5.37 & 30787325 & 44.52 & 95.72 & 90.24 \\
& 7R4347Q2B359PA & 38258824 & 5.74 & 25517669 & 47.45 & 95.91 & 90.64 \\
\multirow{2}{*}{ Total } & 7R4349Q2B360PA & 32355865 & 4.85 & 26705240 & 44.95 & 95.37 & 89.67 \\
\hline
\end{tabular}

GC: Ratio of the sum of guanine and cytosine; Q20: Ratio of bases with a mass value greater than or equal to 20; Q30: Ratio of bases with a mass value greater than or equal to 30 . 
Figure 1. Differential expression genes.

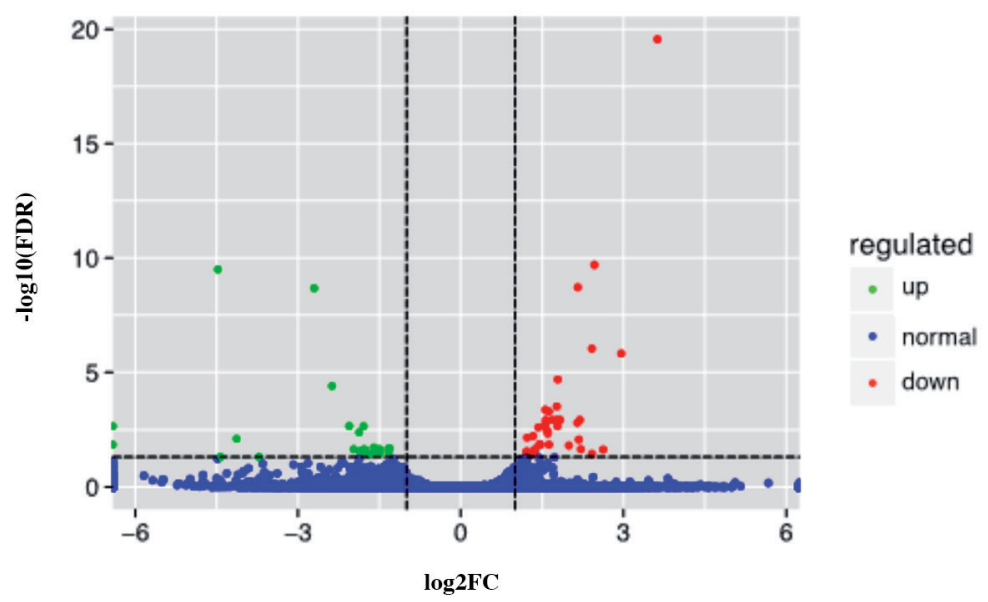

The fold changes (Mutant (Mut)/Wild-type (WT), in logarithm scale) were represented by y-axis (-log 10 of the qvalue). Each dot in the figure represents a single gene, and the green and red dots represent the significantly differential genes. Green dot indicates an up-regulated gene (Mut/WT), and red dot indicates a down-regulated gene (Mut/WT). Blue dots represent genes with nonsignificant difference.

Figure 2. Gene ontology annotation of differentially expressed genes.

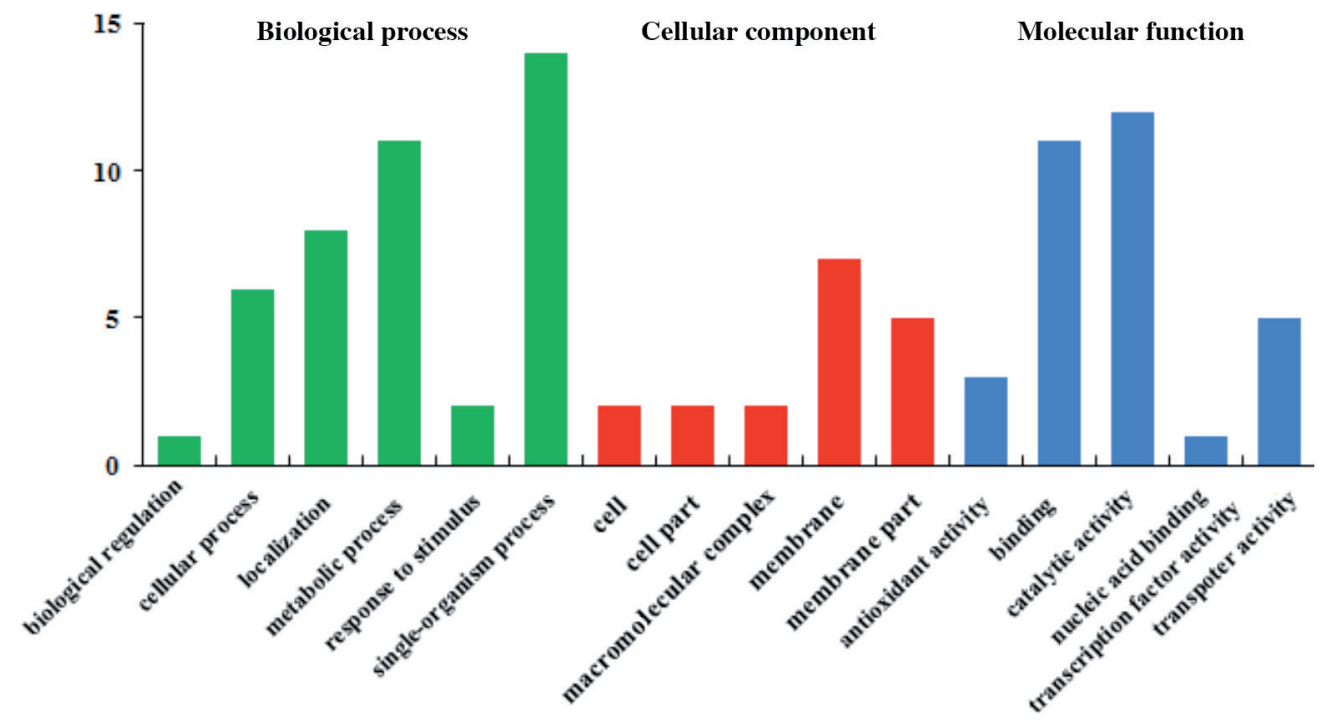

\section{KEGG Pathway analysis of DEGs}

The KEGG enrichment analysis on DEGs showed that, 12 out of the 61 genes were annotated to seven pathways, including the cofactors and vitamin metabolism, amino acid metabolism, lipid metabolism, etc. (Figure 3).

Figure 3. KEGG annotation of differentially expressed genes.

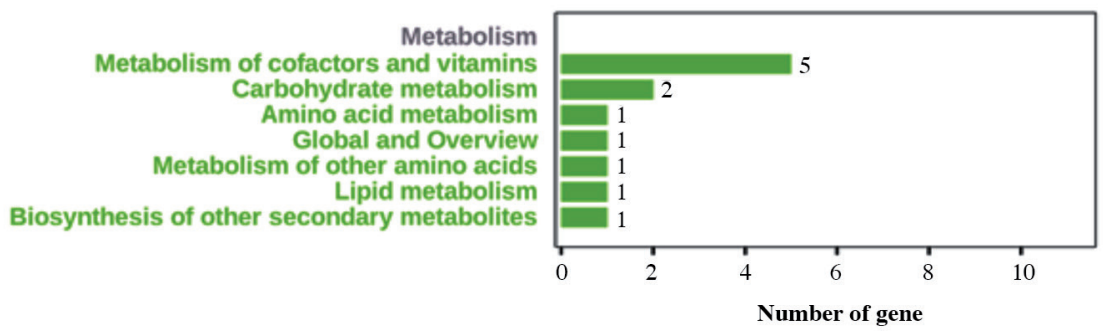

KEGG: Kyoto Encyclopedia of Genes and Genomes. 


\section{Screening of DEGs}

Among the DEGs selected from transcriptome sequencing data, we identified 8 genes related to the protective enzymes and endogenous hormone metabolism, including one auxin-induced protein related gene Cap.ARATH (Capana04g001523), one ethylene response related gene Cap.RAP2 (Capana08g000001), five peroxidase related genes Cap.POD (Capana02g003649, Capana12g002272, Capana01g001329, Capana03g002143, and Capana12g002274), and one MYB family related gene Cap.MYB1R1 (Capana05g002019).

\section{Quantitative verification of the critical genes}

The expression levels of eight genes related to protective enzymes and endogenous hormone metabolism were quantified, and found to be significantly different between mutant and WT (Figure 4). Among them, the expression levels of auxin-induced protein related gene Cap.ARATH (Capana04g001523) and ethylene response related gene Cap.RAP2 (Capana08g000001) were significantly higher in mutant. The peroxidase related genes Cap.POD (Capana12g002272, Capana02g003649, Capana03g002143 and Capana12g002274) were up-regulated in mutants to different extents,

Figure 4. Relative gene expression and FPKM level.
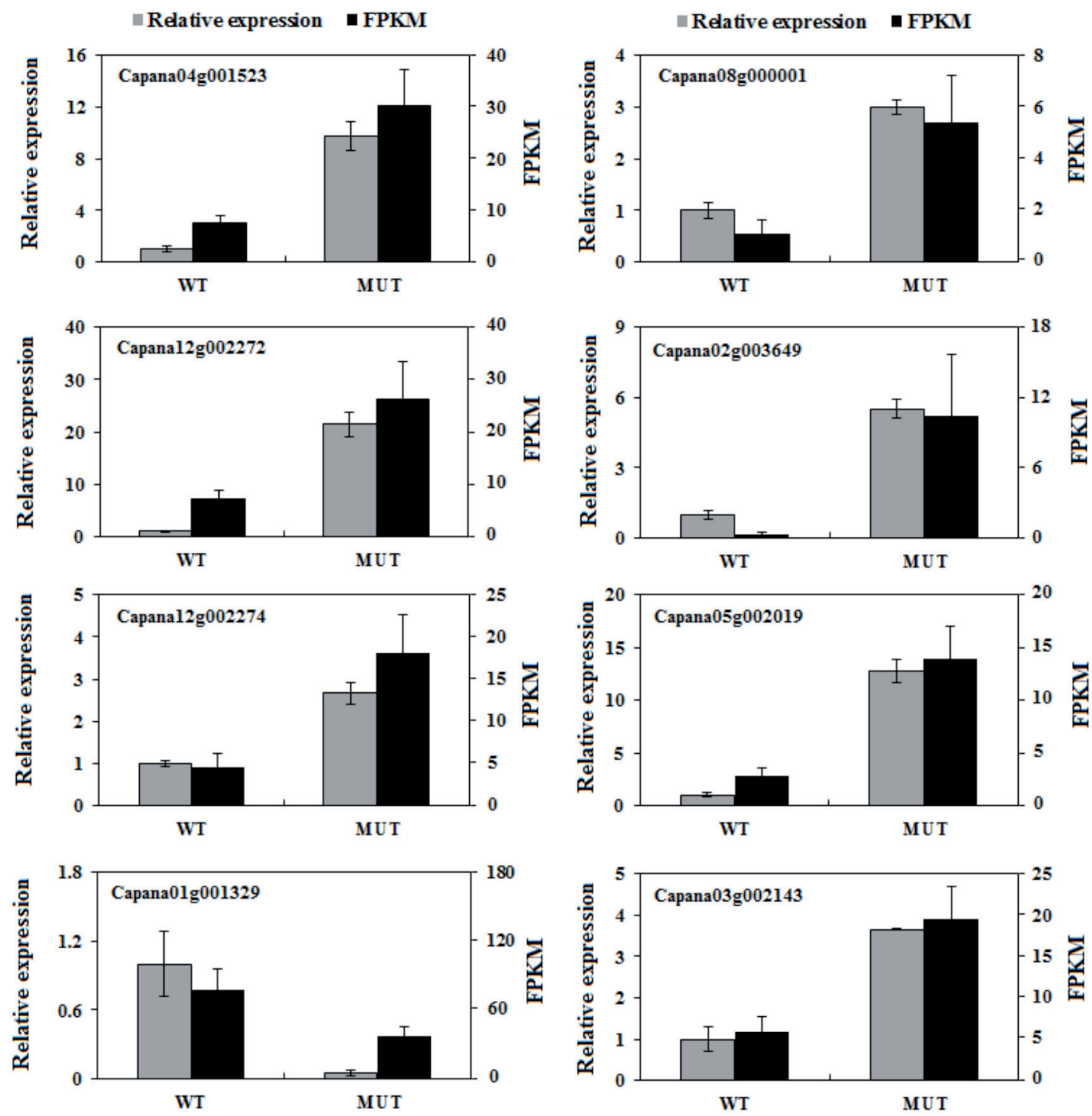

FPKM: Fragments per kilobase of transcript per million fragments mapped; WT: wild-type; Mut: mutant. 
which was consistent with the changes in POD activity; but another peroxidase related gene (Capana01g001329) was significantly down-regulated in mutants compared to WT. The expression level of MYB family related gene Cap.MYBIRI (Capana05g002019) was also significantly increased in mutant.

\section{DISCUSSION}

Numerous studies have shown that waterlogging stress can cause physiological and biochemical changes in plant, leading to inhibited growth and development (Ullanat and Jayabaskaran, 2002; Perata and Voesenek, 2007; Vidoz et al., 2010; Qi et al., 2011). Under waterlogging stress, the root environment easily becomes hypoxic, which hinders the oxygen supply, resulting in suppression of mitochondrial ATP synthesis and NADH oxidation. Thereby the plant cannot maintain energy metabolism through normal aerobic respiration and switch to anaerobic respiration, which costs more organic material, generates less energy, and produces harmful metabolites such as ethanol, leading to metabolic disorders and growth inhibition in plant. MDA and $\mathrm{OH}$ are the lower oxidation products of the free radical chain reaction caused by the continuous accumulation of ROS. Its content reflects the toxic level of ROS to plants, which can deactivate the proteins and biological enzymes on the cell membrane, destroy the structure and function of the biofilm, and affect the normal material metabolism of cells. In this study, we found that the waterlogging resistant mutant exhibited significantly higher activities of antioxidant enzymes and more osmotic regulation substance under waterlogging treatment, as well as less MDA and ${ }^{\circ} \mathrm{OH}$. These results suggest that the waterlogging stress can cause reduced antioxidant enzyme activity and osmotic regulation substance of pepper root system, and the mutants are able to maintain high enzyme activity and osmotic substance content under this condition, in order to resist the waterlogging environment.

The physiological and biochemical changes of plant under waterlogging stress are actually a series of chain changes caused by the altered gene expression in the related metabolic pathways. As a result of these changes, the content of antioxidant enzymes, proline, soluble sugar, glutathione and other metabolites increased, thus enhancing the waterlogging resistance of plants. Zou et al. (2010) used suppression subtractive hybridization (SSH) and found that many genes were upregulated in maize under flooding conditions, and 66 genes were located close to the known quantitative trait locus (QTL). Du et al. (2014; 2016) used reverse genetics and bioinformatics to explore the AP2/ERF and GT transcription factors associated with maize waterlogging resistance. There is also a study showing that SublA gene is a key regulator for waterlogging resistance, and it can effectively reflect ethylene production and response (Damanik et al., 2010)

Figure 5. The mechanism diagram of waterlogging stress in mutant.

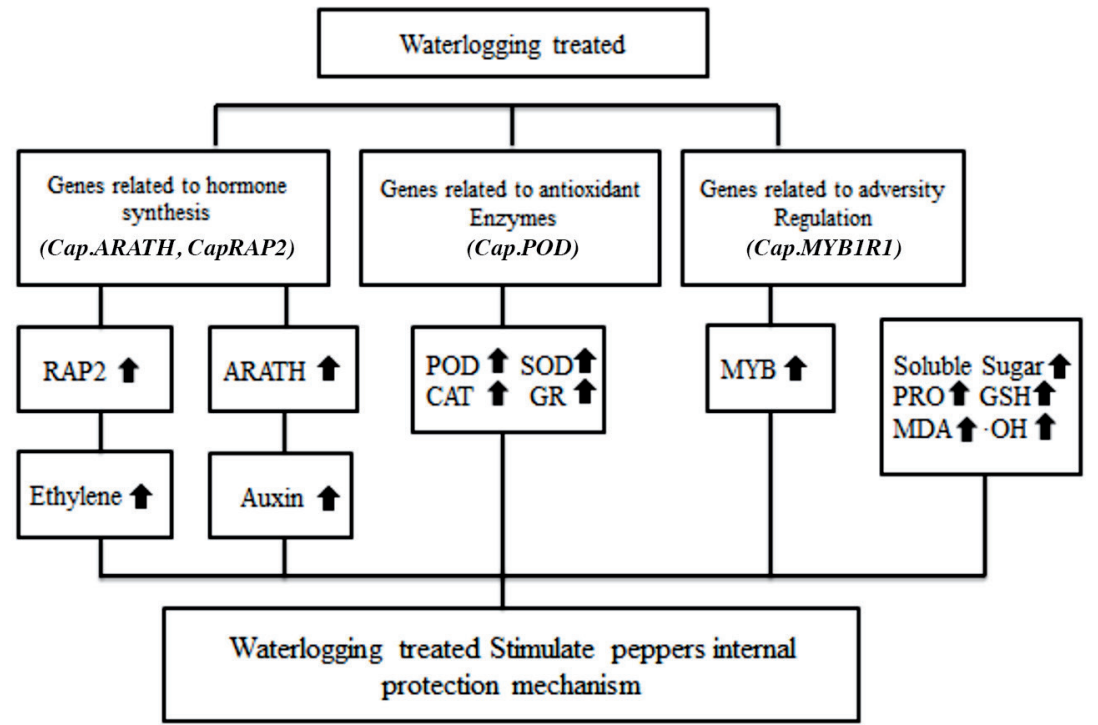

SOD: Superoxide dismutase; POD: peroxidase; CAT: catalase; GR: glutathione reductase; PRO: proline; GSH: glutathione; MDA: malondialdehyde; 'OH: hydroxyl radical. 
through bud elongation and energy consumption (including carbohydrates, fats and proteins) during and after flooding; overexpressing of Sub1A in rice can enhance the waterlogging resistance (Xu et al., 2006; Fukao and Bailey-Serres, 2008). Low oxygen condition promotes the ethylene synthesis in root system (Atwell et al., 1988). For example, under waterlogging stress, tomato can induce adventitious root formation by regulating the expression of ACS, a key gene for ethylene synthesis, through ethylene-responsive elements (ERFs), and promoting the interaction between ethylene and auxin (Vidoz et al., 2010). Hattori et al. (2009) reported that under waterlogging stress, two genes SK1 and SK2 interacted with ethylene-responsive elements (ERFs) in the ethylene signal transduction pathway, thereby triggering the GA involved stem elongation and growth. In this study, transcriptome analysis revealed that the auxin-induced protein related gene Cap.ARATH and ethylene response related gene Cap.RAP2, which can promote auxin and ethylene content, were significantly upregulated in mutant, indicating that the mutations in mutants may be helpful to increase the auxin and ethylene synthesis of plant root system under waterlogging stress, and maintain the normal growth of pepper by increasing the contents of auxin and ethylene.

Studies have shown that MYB transcription factors are related to the plant morphogenesis (Zhang et al., 2009; Brownfield et al., 2009) and metabolic regulation of many primary products and secondary products (Cone et al., 1986; Song et al., 2011; Peng and Moriguchi, 2013; Tombuloglu et al., 2013) such as anthocyanins and phenylpropanoids, which are closely related to plant resistance. In this study, we found that the expression of Cap.MYBIRI was significantly increased in mutants under waterlogging stress. Cap.MYBIR1 is an important transcription factor gene, and its increased expression helps to control the production of related metabolites to enhance the plant waterlogging resistance. Waterlogging can cause the formation of large numbers of aerenchyma in root system, leading to the decrease in plant lignin content (Tao et al., 1998; Chen et al., 2015). Also, certain genes in plant can participate in multiple metabolic regulation pathways (Arce-Rodríguez and Ochoa-Alejo, 2017). Our study found that Capana01g001329 not only participates in the regulation of POD enzyme, but also takes part in the regulation of lignin biosynthesis. The expression of Capana01g001329 in mutants was significantly decreased, which may due to its involvement in regulating lignin synthesis. The reduced root lignin causes regular separation and differentiation of root cells or programmed cell death to form hollow cavities, which, to some extent, promotes the aerenchyma formation in plant root and enhances the waterlogging resistance.

$\mathrm{Xu}$ et al. (2017b) found that in cucumber, the GO terms 'response to chemical stimulus', 'oxidoreductase activity', 'regulation of cellular process' and 'regulation of metabolic process' were highly enriched after waterlogging stress. Qi et al. (2012) and Christianson et al. (2010) analyzed the differentially expressed genes of cucumber and cotton roots after waterlogging, and found that the differential genes in cucumber were mainly involved in C cycle, photosynthesis, generation and elimination of ROS, and hormone-mediated signaling pathways, while the differential genes of cotton were mainly involved in cell wall growth, fermentation pathway, hormone signal response and C-N metabolism, In this study, the transcriptome sequencing also identified several genes associated with single-organism processes, metabolic processes, and catalytic processes, etc.; indicating that different plants had different waterlogging resistant mechanisms. Therefore, the DEGs we identified by comparing pepper mutants and wild-type after waterlogging stress provide important information for understanding the waterlogging damage and resistance mechanism of pepper.

\section{CONCLUSIONS}

In conclusion, we proposed a model of the protective mechanism of mutant pepper against waterlogging stress. Waterlogging stress can induce the synthesis of related hormones and altered expression of antioxidant enzymes in root system, leading to the changes in some endogenous hormones and protective enzymes, which cause the growth inhibition and even plant death. However, the waterlogging resistance mutants can effectively up-regulate the expression of Cap. ARATH, Cap.RAP2, Cap.MYBIR1, and Cap.POD expression thus increasing the activities of endogenous hormones (ethylene and auxin), antioxidant enzymes (peroxidase, superoxide dismutase, catalase, glutathione reductase) and content of related inclusions (soluble sugar, proline and glutathione) under waterlogging stress and maintaining the plant growth and development. 


\section{ACKNOWLEDGEMENTS}

This work was supported by Hunan Provincial Natural Science Foundation of China (Nr 2017JJ2147). The data sets supporting the results of this article are available in the NCBI Sequence Read Archive (SRA) repository (https://www. ncbi.nlm.nih.gov/sra/?term=SRP136291).

\section{REFERENCES}

Arce-Rodríguez, M.L., and Ochoa-Alejo, N. 2017. An R2R3-MYB transcription factor in capsaicinoid biosynthesis. Plant Physiology 174:1359-1370.

Atwell,B.J.,Drew,M.C., and Jackson,M.B.1988. The influence of oxygen deficiency on ethylene synthesis, 1-aminocyclopropane1-carboxylic acid levels and aerenchyma formation in roots of Zea mays. Physiologia Plantarum 72:15-22.

Brownfield, L., Hafidh, S., Borg, M., Sidorova, A., Mori, T., and Twell, D. 2009. A Plant germline-specific integrator of sperm specification and cell cycle progression. Plos Genetics 5:e1000430.

Chen, Y.S., Ying, X.C., Shen, P.F., Xu, Y.R., Zu, W.G., and Shan, Y.H. 2015. Difference of decay process of cellulose and lignin in wheat straw. Journal of Yangzhou University 36:68-72.

Christianson, J.A., Llewellyn, D.J., Dennis, E.S., and Wilson, I.W. 2010. Global gene expression responses to waterlogging in roots and leaves of cotton (Gossypium hirsutum L.) Plant and Cell Physiology 51:21-37.

Cone, K.C., Burr, F.A., and Burr, B. 1986. Molecular analysis of the maize anthocyanin regulatory locus C1. Proceedings of the National Academy of Sciences of the United States of America 83:9631-9635.

Damanik, R.I., Maziah, M., Ismail, M.R., Ahmad, S., and Zain, A.M. 2010. Responses of the antioxidative enzymes in Malaysian rice (Oryza sativa L.) cultivars under submergence condition. Acta Physiologiae Plantarum 32:739-747.

Du, H.W., Huang, M., and Liu, L. 2016. The genome wide analysis of GT transcription factors that respond to drought and waterlogging stresses in maize. Euphytica 208:113-122.

Du, H.W., Huang, M., Zhang, Z.X., and Cheng, S.Y. 2014. Genome-wide analysis of the AP2/ERF gene family in maize. Euphytica 198:115-126.

Fukao, T., and Bailey-Serres, J. 2008. Submergence tolerance conferred by SublA is mediated by SLR1 and SLRL1 restriction of gibberellin responses in rice. Proceedings of the National Academy of Sciences of the United States of America 105:16814-16819.

Hattori, Y., Nagai, K., Furukawa, S., Song, X.J., Kawano, R., Sakakibara, H., et al. 2009. The ethylene response factors SNORKEL1 and SNORKEL2 allow rice to adapt to deep water. Nature 460:1026-1030.

Jin, J., Zhu, C., Fu, Y.P., and Sun, Z.X. 2003. Screen of rice mutants in adversity of low-temperature during germination. Journal of Zhejiang Agricultural University (Agriculture and Life Sciences) 29:207-209.

Lee, Y.H., Kim, K.S., Jang, Y.S., Hwang, J.H., Lee, D.H., and Choi, I.H. 2014. Global gene expression responses to waterlogging in leaves of rape seedlings. Plant Cell Reports 33:289.

Liu, Z.B., Zhang, Y.P., Ou, L.J., Kang, L.Y., Liu, Y.H., Lv, J.H., et al. 2017. Identification and characterization of novel microRNAs for fruit development and quality in hot pepper (Capsicum annuит L.) Gene 608:66-72.

Ou, L.J., Chen, B., and Zou, X.X. 2012. Effects of drought stress on photosynthesis and associated physiological characters of pepper. Acta Ecologica Sinica 32:2612-2619.

Ou, L.J., Dai, X.Z., Zhang, Z.Q., and Zou, X.X. 2011. Responses of pepper to waterlogging stress. Photosynthetica 49:339-345.

Pei, C.M., Zhang, Z.Y., and Jin, M.A. 2017. Identification and characterization of salt-responsive transcription factors in leaves of southern type alfalfa. Molecular Plant Breeding 15:315-321.

Peng, T., and Moriguchi, T. 2013. The molecular network regulating the coloration in apple. Scientia Horticulturae 163:1-9.

Perata, P., and Voesenek, L.A.C.J. 2007. Submergence tolerance in rice requires Sub1A, an ethylene-response-factor-like gene. Trends in Plant Science 12:43-46.

Qi, X.H., Li, T., Xu, Q., and Chen, X.H. 2011. Modulation of chlorophyll contents and anti-oxidant systems in two cucumber varieties under waterlogging stress and subsequent drainage. Journal of Pomology and Horticultural Science 86:337-342.

Qi, X.H., Xu, X.W., Lin, X.J., Chen, X.H., and Zhang, W.J. 2012. Identification of differentially expressed genes in cucumber (Cucumis sativus L.) root under waterlogging stress by digital gene expression profile. Genomics 99:160-168.

Song, S., Qi, T., Huang, H., Ren, Q., Wu, D., Chang, C., et al. 2011. The jasmonate-ZIM domain proteins interact with the R2R3-MYB transcription factors MYB21 and MYB24 to affect jasmonate-regulated stamen development in Arabidopsis. Plant Cell 23:1000-1013.

Tan, C., Liu, Z., Huang, S., Li, C., Ren, J., Tang, X., et al. 2018. Pectin methylesterase inhibitor (PMEI) family can be related to male sterility in Chinese cabbage (Brassica rapa ssp. pekinensis). Molecular Genetics and Genomics 293(2):343-357.

Tao, R.Z., Fei, B.H., Liao, Y.F., and Zhang, J. 1998. Effects of intermittent flood on pulp marking and papermaking properties of Taxodium ascendens wood. Journal of Anhui Agricultural University 2:109-111. 
Tombuloglu, H., Kekec, G., Sakcali, M.S., and Unver, T. 2013. Transcriptome-wide identification of R2R3-MYB transcription factors in barley with their boron responsive expression analysis. Molecular Genetics and Genomics 288:141-155.

Ullanat, R., and Jayabaskaran, C. 2002. Distinct light-, cytokinin- and tissue-specific regulation of calcium-dependent protein kinase gene expression in cucumber (Cucumis sativus). Plant Science 162:153-163.

Valliyodan, B., Ye, H., Song, L., Murphy, M., Shannon, J.G., and Nguyen, H.T. 2017. Genetic diversity and genomic strategies for improving drought and waterlogging tolerance in soybeans. Journal of Experimental Botany 68:1835-1849.

Vidoz, M.L., Loreti, E., Mensuali, A., Alpi, A., and Perata, P. 2010. Hormonal interplay during adventitious root formation in flooded tomato plants. Plant Journal 63:551-562.

Wu, C.J., Bordeos, A., Madamba, M.R., Baraoidan, M., Ramos, M., Wang, G.L., et al. 2008. Rice lesion mimic mutants with enhanced resistance to diseases. Molecular Genetics and Genomics 279:605-619.

Xu, X., Chen, M., Ji, J., Xu, Q., Qi, X., and Chen, X. 2017b. Comparative RNA-seq based transcriptome profiling of waterlogging response in cucumber hypocotyls reveals novel insights into the de novo adventitious root primordia initiation. BMC Plant Biology 17(1):129.

Xu, K.N., Xu, X., Fukao, T., Canlas, P., Maghirang-Rodriguez, R., Heuer, S., et al. 2006. SublA is an ethylene-response-factorlike gene that confers submergence tolerance to rice. Nature 442:705.

Xu, T.T., Yu, N., Zhang, Y.X., Bi, Z.Z., Wu, W.X., Cao, Y.Y., et al. 2017a. Identification of rice blast resistance mutant $l m m 326$ and preliminary analysis of its regulatory pathway. Chinese Journal of Rice Science 31:335-344.

Yang, B.Z., Liu, Z.B., Zhou, S.D., Ou, L.J., Dai, X.Z., Ma, Y.Q., et al. 2016. Exogenous $\mathrm{Ca}^{2+}$ alleviates waterlogging-caused damages to pepper. Photosynthetica 54(4):1-11.

Zhang, Y., Cao, G., Qu, L.J., and Gu, H. 2009. Characterization of Arabidopsis MYB transcription factor gene AtMYB17 and its possible regulation by LEAFY and AGL15. Journal of Genetics and Genomics 36:99-107.

Zhang, M., Yang, Y., and Kang, Z.R. 2011. A preliminary study on drought-resistant mutant induction in southern highbush blueberry plants with EMS mutagenesis. Journal of Southwest China Normal University 36:132-137.

Zou, X.X. 2009. Pepper genetic and breeding. Science Press, Beijing, China.

Zou, X.L., Jiang, Y.Y., Liu, L., Zhang, Z.X., and Zheng, Y.L. 2010. Identification of transcriptome induced in roots of maize seedlings at the late stage of waterlogging. BMC Plant Biology 10:189.

Zou, X., Tan, X., Hu, C., Zeng, L., Lu, G., Fu, G., et al. 2013. The transcriptome of Brassica napus L. roots under waterlogging at the seedling stage. International Journal of Molecular Sciences 14:2637. 